\title{
Editorial
}

\section{Modeling and Control of Complex Dynamic Systems: Applied Mathematical Aspects}

\author{
Zhiwei Gao, ${ }^{1}$ Dexing Kong, ${ }^{2}$ and Chuanhou Gao ${ }^{2}$ \\ ${ }^{1}$ Division of Electric Engineering and Physics, Faculty of Engineering and Environment, \\ Northumbria University, Newcastle upon Tyne NE1 8ST, UK \\ ${ }^{2}$ Department of Mathematics, College of Science, Zhejiang University, Hang Zhou 310027, China
}

Correspondence should be addressed to Zhiwei Gao, zhiwei.gao@northumbria.ac.uk

Received 25 November 2012; Accepted 25 November 2012

Copyright (c) 2012 Zhiwei Gao et al. This is an open access article distributed under the Creative Commons Attribution License, which permits unrestricted use, distribution, and reproduction in any medium, provided the original work is properly cited.

\section{Introduction}

The concept of complex dynamic systems arises in many varieties, including the areas of energy generation, storage and distribution, ecosystems, gene regulation and health delivery, safety and security systems, telecommunications, transportation networks, and the rapidly emerging research topics seeking to understand and analyse. Such systems are often concurrent and distributed, because they have to react to various kinds of events, signals, and conditions. They may be characterized by a system with uncertainties, time delays, stochastic perturbations, hybrid dynamics, distributed dynamics, chaotic dynamics, and a large number of algebraic loops. This special issue provides a platform for researchers to report their recent results on various mathematical methods and techniques for modelling and control of complex dynamic systems and identifying critical issues and challenges for future investigation in this field. This special issue amazingly attracted one-hundred-andeighteen submissions, and twenty-seven of them are selected through a rigorous review procedure.

The selected papers contribute mathematical modelling, parameter identification, monitoring and diagnosis, optimization, and control for a variant of complex systems such as chaotic systems (4 papers), impulsive and singular systems (4 papers), nonlinear systems (12 papers), delay systems (3 papers), stochastic systems (3 papers), and gene complex network (1 paper). From the viewpoint of modelling, the related papers mainly investigate mathematical modelling for complex systems by using physical laws or deal with model identification by using the data based training and estimation. Along with the dynamic 
equation models, graph-based model is of interest for modelling complex gene regulation network. From the viewpoint of control, the adopted techniques mainly include adaptive control, impulsive control, sliding mode control, fuzzy control, nonlinear optimization, and optimal control. From the view of application, the involved systems include mechanical dynamic systems, biological systems, aerospace systems, fluid dynamic systems, energy systems, and ecological-economic systems.

We are going to introduce the selected papers in the categories of chaotic systems, impulsive and singular systems, nonlinear systems / processes, time-delay systems, stochastic systems, and gene complex network.

\section{Modelling and Control for Chaotic Systems}

For modelling and control of chaotic systems, the paper entitled "The chaotic prediction for aeroengine performance parameters based on nonlinear PLS regression" is contributed by C. Zhang and $\mathrm{J}$. Yue. On the basis of the actual air-borne data of the aircraft communications addressing and reporting system, a nonlinear partial least square regression method is proposed to predict chaotic function of the engine exhaust temperature time series of the aero-engine, which will benefit the condition monitoring and diagnosis for aero engines.

The paper entitled "Dynamic analysis of a hybrid squeeze film damper mounted rub-impact rotor-stator system," authored by C. C.-Jian, presents a detailed analysis on dynamic behaviour of a rotor-stator system. It reveals that its dynamic behaviours may suddenly evolve into chaos from periodic motions without any transition. The presented results provide useful insights into the design and development of a rotor-bearing complex system.

The paper entitled "Optimal control for a class of chaotic systems," authored by J. Zhang and W. Tang, presents an optimal state-feedback control strategy for a class of chaotic systems. The system considered is transformed into a set of uncertain piecewise linear systems, and an optimal robust controller is designed by solving an optimisation problem under the constraints of nonlinear inequalities.

In the paper entitled "Bifurcation analysis for a kind of nonlinear finance system with delayed feedback and its application to control of chaos," R. Zhang presents a quantitative distribution analysis on the roots of the associate characteristic equation for a kind of nonlinear finance systems with time-delay feedback. The conditions of ensuring the existence of Hopf bifurcation are addressed and the explicit formulas are derived for determining the stability and direction of the bifurcating periodic solutions.

\section{Modelling and Control of Impulsive and Singular Systems}

For modelling and control of impulsive and singular systems, the paper entitled "The analysis and control for singular ecological-economic model with harvesting and migration" is contributed by Q. Zhang et al. This paper presents a singular ecological-economic model for the populations with harvesting and migration. The local stability and dynamic behaviour of the model are investigated. By transforming the singular model into a single-input single-output model, variable structure control is applied to eliminate the complex behaviours of the considered problem.

The paper entitled "Multi-state dependent impulsive control for pest management," authored by $\mathrm{H}$. Cheng et al., presents a combined control strategy for pest management. The combined control strategy adopts different control methods according to different thresholds. 
The existence and convergence of the first-order periodic solution of such systems are further analysed.

The paper entitled "Dynamic analysis of a predator-prey (Pest) model with disease in prey and involving an impulsive control strategy," is contributed by $\mathrm{M}$. Zhao et al., which presents a dynamic analysis of a predator-prey (pest) model with disease in prey. The model involves an impulsive control strategy to release infected prey at fixed times. Theoretical analysis on the local asymptotical stability and global attractivity for the semitrivial periodic solution is also presented.

In paper entitled "A matrix method for determining eigenvalues and stability of singular neutral delay-differential systems," J. Ma et al. present an analysis on the eigenvalues and stability of singular neutral delay-differential systems. The matrix pencil and linear operator methods are used to derive a new algebraic criterion to obtain imaginary axis eigenvalues. In addition, a criterion for the asymptotic stability is introduced.

\section{Modelling and Control of Nonlinear Systems/Processes}

In the paper entitled "Adaptive fault detection for complex dynamic processes based on jit updated data set," J. Li et al. presents a fault detection technique for nonlinear complex industrial processes. Just-in-time and k-nearest neighbour methods are integrated to construct a flexible and adaptive detection scheme. The updating of the database is taken into account in the design of fault detection algorithm.

The paper, "Computation of the added masses of an unconventional airship," authored by N. Azouz et al., presents a modelling method for an unmanned airship. An aerodynamic investigation is firstly presented and a mathematical analysis of the velocity potential flow of the shape of the airship is developed. The whole system, described by nonlinear equations subjected to boundary conditions, governing the interaction air-structure, is solved in an analytical setting.

In the paper entitled "Nonlinearities in drug release process from polymeric microparticles: long-time-scale behavior," E. S. Bacaita et al. present a theoretical model of the drug release process from polymeric microparticles through dispersive fractal approximation of motion. The drug release process takes place through cnoidal oscillations modes of a normalized concentration field, which indicates that the drug particles assemble in a lattice of nonlinear oscillators occur macroscopically.

The paper "Application of the poor man's navier-stokes equations to real-time control of fluid flow," authored by J. M. Polly and J. M. McDonough, presents an application study of the Poor Man's Navier Stokes (PMNS) equations to real time control of fluid flow. The PMNS equations comprise a discrete dynamic system that is algebraic so that they can be easily and rapidly solved. Theoretical analysis of the PMNS equations is discussed and a control force is added to control the fluid flow.

In the paper entitled "Data fusion based hybrid approach for the estimation of urban arterial travel time," S. P. Anusha et al. present a data based approach to estimate the travel time in urban arterials in India. Three different approaches are proposed, namely, highway capacity manual based method, Kalman filter based method and hybrid method.

In the paper entitled, "Adaptive control of a two-item inventory modelwith unknown demand rate coefficients," Alshamrani presents a multiitem inventory model with unknown demand coefficients. The output of the system is tracked by an adaptive control approach with a nonlinear feedback. The asymptotic stability of the adaptive controlled system is proven by the Lyapunov technique. 
In the paper entitled, "Optimal control of a spatio-temporal model for malaria: synergy treatment and prevention," M. Zorom et al. present a metapopulation model for malaria with two control variables, treatment and prevention, distributed between $n$ different patches. A mathematical model with three patches is constructed using optimal control techniques. Based on the model, qualitative suggestions for treatment and prevention of Malaria are provided.

In the paper entitled "Nonsmooth recursive identification of sandwich systems with backlashlike hysteresis," R. Dong et al. present a recursive gradient identification algorithm based on the bundle method for sandwich systems with backlash-like hysteresis. A dynamic parameter estimation scheme is developed to handle the nonsmooth problem caused by the backlash embedded in the system. The bundle method is used to estimate the search direction of the subgradient algorithm.

In the paper entitled "The hybrid adaptive control of T-S fuzzy system based on niche," T. Zhao et al. presents a hybrid adaptive fuzzy control method with the function of continuous supervisory control. The method is based on the niche characteristics, whose control law is designed by tracking, continuous supervisory, and adaptive compensation. An adaptive compensator and adaptive laws of parameters are designed to embody system adaptability.

The paper entitled "Stability and limit oscillations of a control event-based sampling criterion," authored by M. De La Sen et al., investigates the presence of limit oscillations in an adaptive sampling system. An extended sampling criterion is proposed and the limit oscillations are interpreted on the basis of adaptive sampling in nonlinear dynamic systems.

In the paper entitled "Modeling optimal scheduling for pumping system to minimize operation cost and enhance operation reliability," Y. Luo et al. present an optimal scheduling plan for pumping system to minimize operation cost and enhance operation reliability. The operation reliability is explored by investigating vibration level and a model is built as a function of the capacity and rotation speed of the pump for the operation reliability.

In the paper entitled "Improving the solution of least squares support vector machines with application to a blast furnace system," L. Jian et al. present a strategy to speed up the search of the solution of the least square support vector machine (LSSVM) by using minimal residual method to solve saddle point systems in LSSVM directly. The proposed method is validated by a blast furnace industrial process.

\section{Modelling and Control of Systems with Time Delays}

In the paper, entitled "Modelling and analysis of epidemic diffusion within small-world network," M. Liu and Y. Xiao present two different models, Susceptible-Exposure-Infected-RecoveredSusceptible model and Susceptible-Exposure-Infected-Quarantine-Recovered-Susceptible model, to describe the rule of epidemic diffusion. The models are analysed within the smallworld network. Theoretical properties such as condition for occurrence of disease diffusion, existence, and global stability of the disease free equilibrium are addressed.

In the paper entitled "Synchronization of coupled networks with mixed delays by intermittent control," the synchronization of coupled networks with mixed delay is investigated by employing Lyapunov functional method and intermittent control. A sufficient condition is derived to ensure the global synchronization of coupled networks and an intermittent controller is designed to control the system.

In the paper entitled "Type-K exponential ordering with application to delayed hopfieldtype neural networks," B.-W. Wang presents order-preserving and convergent results for delay functional differential equations without quasi-monotone condition under type-K 
exponential ordering. The delayed Hopfield-type neural networks with a type $\mathrm{K}$ monotone interconnection matrix are considered as an application and the attractor is obtained.

\section{Modelling and Control of Stochastic Systems}

For modelling and control of stochastic systems, the paper entitled "Pareto design of decoupled sliding-mode controllers for nonlinear systems based on a multiobjective genetic algorithm," authored by M. J. Mahmoodabadi et al., presents the pareto design of decoupled sliding-mode controller based on multiobjective genetic algorithm for several fourth-order coupled nonlinear systems. The decoupled sliding mode controller is applied to stabilize the fourthorder coupled systems at the equilibrium point. The optimal parameters of the decoupled sliding-mode controller are obtained using the multiobjective genetic algorithm.

In the paper entitled "Mean square almost periodic solutions for impulsive stochastic differential equations with delays," R. Zhang et al. present a result on existence and uniqueness of mean square almost periodic solutions for a class of impulsive stochastic differential equations with delay. This is an extension of the earlier works reported in the literature.

In the paper entitled "Bank liquidity and the global financial crisis," F. Gideon et al. present an analysis on the stochastic dynamics of bank liquidity parameters such as liquid assets and net cash outflow in the case of global financial crisis. Numerical results relating to bank behaviour are obtained and a theoretical-quantitative approach to bank liquidity provisioning is provided.

\section{Modelling and Regulation of Gene Complex Network}

The paper entitled "Modules identification in gene positive networks of hepatocellular carcinoma using pearson agglomerative method and pearson cohesion coupling modularity" is contributed by J. Hu and Z. Gao. The paper firstly addresses the concepts of gene network community and gene positive network on the basis of weighted undirected graphs. A module identification algorithm is developed to calculate the threshold values of gene positive networks. The fourteen strong modules and thirteen very strong modules are obtained and the relations between these modules are analysed. The biological significance of these modules is explained. The research may provide new clues and ideas for liver cancer treatment.

The special issue involves a variety of research issues for complex systems, which provides a platform for the research community to share their knowledge and experience in complex systems modelling, dynamic analyses, optimization, and control.

\section{Acknowledgment}

Finally the guest editors would like to take this opportunity to thank all the contributions from the authors, reviewers, and journal editorial staff. 


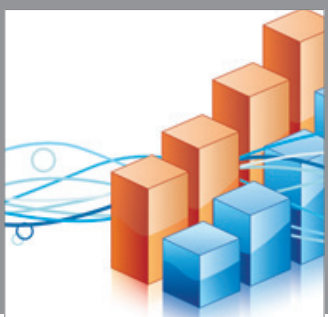

Advances in

Operations Research

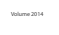

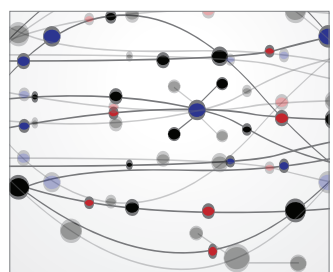

\section{The Scientific} World Journal
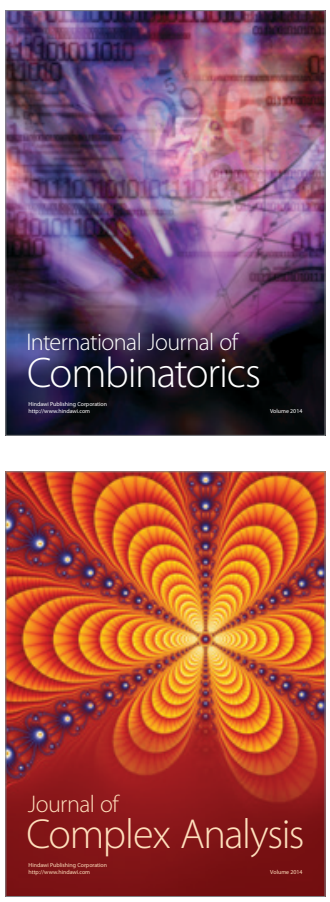

International Journal of

Mathematics and

Mathematical

Sciences
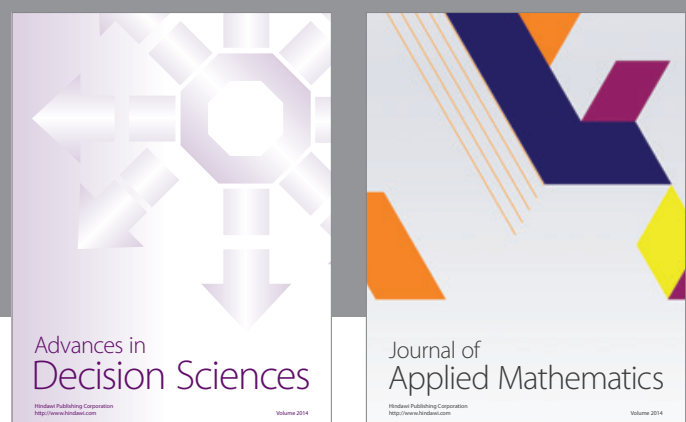

Journal of

Applied Mathematics
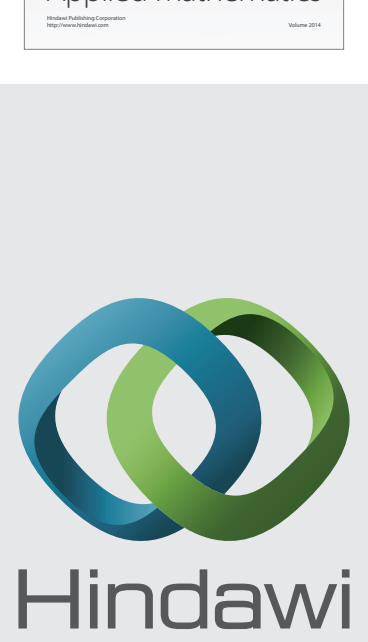

Submit your manuscripts at http://www.hindawi.com
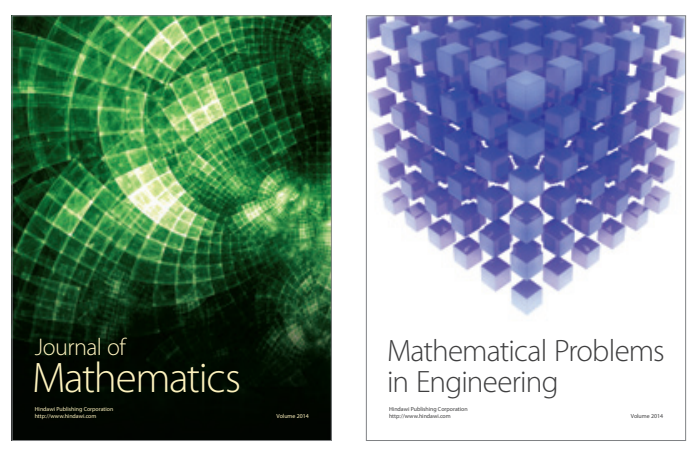

Mathematical Problems in Engineering
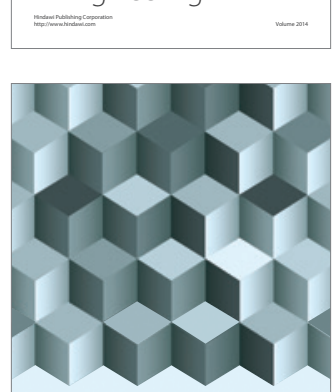

Journal of

Function Spaces
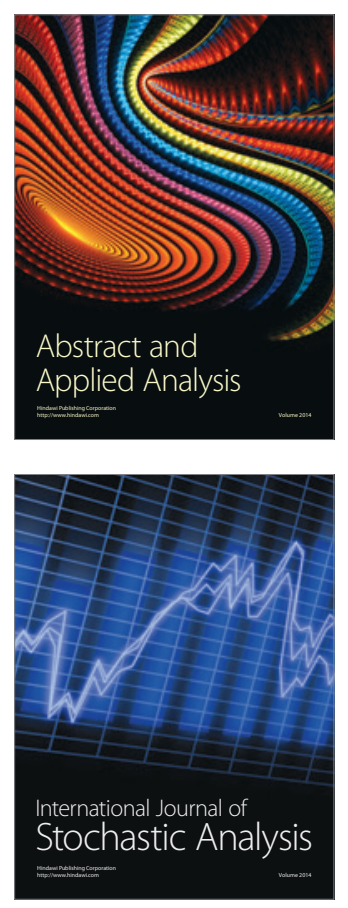

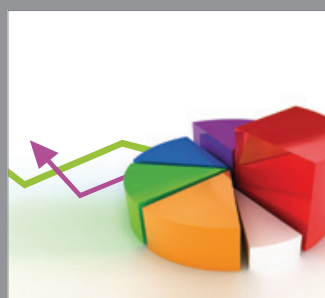

ournal of

Probability and Statistics

Promensencen
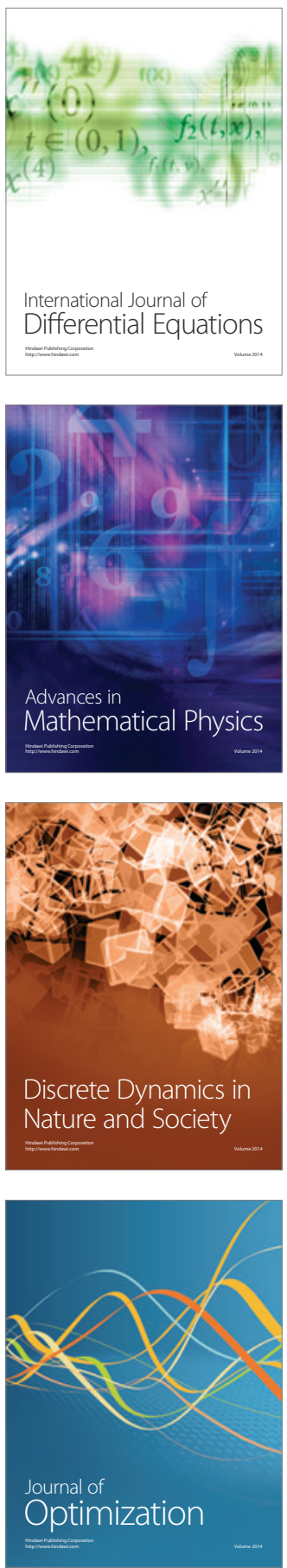